\title{
ROLE OF SERUM CRP AND SERUM LDH IN PREDICTING SEVERITY OF PANCREATITIS- A PROSPECTIVE STUDY
}

\author{
Archana Chilakala1 ${ }^{1}$ S. Fasiulla², Vijay Krishnamurthy ${ }^{3}$
}

${ }^{1}$ Assistant Professor, Department of General Surgery, Sri Venkateswara Medical College (SVMC), Tirupati, Andhra Pradesh, India. ${ }^{2}$ Postgraduate Student, Department of General Surgery, Sri Venkateswara Medical College (SVMC), Tirupati, Andhra Pradesh, India. ${ }^{3}$ Assistant Professor, Department of Anesthesiology, Sri Venkateswara Medical College (SVMC), Tirupati, Andhra Pradesh, India.

\section{ABSTRACT}

\section{BACKGROUND}

Acute pancreatitis is a common disease with a wide clinical variation and its incidence is increasing. ${ }^{1,2,3}$ About $25 \%$ of patients with acute pancreatitis develop the severe form. The average mortality rate in severe acute pancreatitis is about $2-10 \%{ }^{4} \mathrm{New}$ serum markers have recently emerged and their potential for rapid scoring of the disease severity in the acute phase are evaluated and some of them might be used as potential therapeutic targets.

\section{METHODS}

This is a prospective analytical study. Patients more than 18 years of age, of both sexes, with elevated serum pancreatic enzymes, diagnosed of acute pancreatitis and confirmed with imaging studies were included in the study. Patients who had chronic pancreatitis, irrespective of age and sex were excluded.

\section{RESULTS}

Serum CRP and serum LDH levels tend to have an excellent accuracy in predicting the severity in acute pancreatitis.

\section{CONCLUSIONS}

Acute pancreatitis is a condition associated with high morbidity and mortality. The sensitivity and specificity of both serum CRP and LDH were excellent in predicting the severity of acute pancreatitis even on day 1 on par with traditional scoring systems. Both LDH and CRP are simple, easily available, economical biochemical parameters to predict the severity of acute pancreatitis in the early phase of disease.

\section{KEY WORDS}

Serum CRP, Serum LDH, Acute Pancreatitis, Serum Amylase, Serum Lipase

HOW TO CITE THIS ARTICLE: Chilakala A, Fasiulla S, Krishnamurthy V. Role of serum CRP and serum LDH in predicting severity of pancreatitis- a prospective study. J. Evolution Med. Dent. Sci. 2019;8(30):2375-2379, DOI: 10.14260/jemds/2019/520

\section{BACKGROUND}

Acute pancreatitis is a common disease with a wide clinical variation and its incidence is increasing.1,2,3 About $25 \%$ of patients with acute pancreatitis develop the severe form. The average mortality rate in severe acute pancreatitis is about 2 $10 \%{ }^{4}$ Severe acute pancreatitis occurs in two phases.

The "first or early phase" is characterised by extensive pancreatic inflammation and/or necrosis followed by a systemic inflammatory response syndrome (SIRS) that leads to multiple organ dysfunction syndrome (MODS) within the first week. About $50 \%$ of deaths occur within the first week of the attack, mostly from MODS, accounting for nearly 40-60\% of in-hospital deaths in all age groups.5,6,7 The mortality rate associated with MODS is between $30-100 \%$. Infection is not a feature of the early phase. Pro inflammatory cytokines contribute to respiratory, renal, and hepatic failure. The "second or late phase" which starts 14 days after the onset of the disease, is marked by infection of the gland, necrosis and

'Financial or Other Competing Interest': None.

Submission 10-05-2019, Peer Review 09-07-2019,

Acceptance 17-07-2019, Published 29-07-2019.

Corresponding Author:

Dr. Archana Chilakala,

Assistant Professor, Department of General Surgery,

Sri Venkateswara Medical College and

SVRRGG Hospital, Tirupati-517501, Andhra Pradesh, India.

E-mail: archana.chilakala@gmail.com

DOI: $10.14260 /$ jemds $/ 2019 / 520$

\section{(c) (1) $(9)$}

systemic complications causing a significant increase in mortality. Respiratory failure is the most common type of organ failure in acute pancreatitis.

There is a wide variation in the severity of acute pancreatitis. Mild acute pancreatitis undergoes spontaneous remission, but once acute pancreatitis becomes severe, it poses a great risk of death from fatal complications, such as circulatory failure, multi organ failure, and infections.

The assessment of severity during the initial examination is extremely important to improve the survival rate in patients with acute pancreatitis. This is to ensure the quick and accurate diagnosis of severe cases (Which have a high mortality rate), to commence appropriate initial treatment, and, if necessary, to transfer the patient to an advanced specialist medical institution. It is also important to monitor the effects of treatment by serial assessments of severity.

When deciding on an appropriate system of criteria to assess the severity of pancreatitis, it is important to establish whether the assessment (1) depends on an examination method that is easily available at most of the institutions, (2) can be used shortly after admission to the hospital, (3) can be used to monitor the course of the disease and (4) can be performed repeatedly. Ideally it should have high sensitivity and positive prognostic value, and diagnose pancreatic necrosis early (During the first 48 hours). The test should also be readily available in every clinical laboratory, reproducible and cost effective. 
Criteria of severity, such as Ranson's, Glasgow, and Acute Physiology and Chronic Health Evaluation (APACHE) scores have been used for a long time.8,9,10 These scores assess the multiple organ dysfunction induced by the disease and consequently, the greater the number of organs injured, the greater the score.

New serum markers have recently emerged and their potential for providing additional information on the severity of the disease is currently being evaluated. When seeking medical attention (Usually 12 to $24 \mathrm{~h}$ after the onset of pain) most patients do not exhibit multiple organ dysfunction, which emerges by the second or third day and, on admission, numerous mediators can be detected in serum. If the concentration of these biologic factors is correlated to the severity of the disease, and if they are detected before the occurrence of multiple organ dysfunction it is then conceivable that the therapeutic antagonism of these mediators might prevent or attenuate the severity of the multiple organ dysfunction, and consequently the outcome of the disease. These new factors might be important for the rapid scoring of the disease severity in the acute phase and some of them might be used as potential therapeutic targets.

\section{Aim of The Study}

To assess the predictive value of serum CRP and serum LDH in predicting severity of acute pancreatitis.

\section{Definition}

Acute pancreatitis is an inflammatory process which occurs in a normal organ and it is associated with varying involvement of other regional tissues or remote organ systems. Acute pancreatitis is best defined clinically by a patient presenting with 2 of the following 3 criteria

- Characteristic abdominal pain.

- Biochemical evidence of pancreatitis (i.e., amylase or lipase serum levels more than 3 times the upper reference limit)

- Radiologic evidence of pancreatitis on abdominal imaging usually using CT or MRI.

Pancreatitis is classified as acute unless there are computed tomography (CT) or endoscopic retrograde cholangiopancreatography (ERCP) findings of chronic pancreatitis. Any episode of "acute pancreatitis" is then considered to be an exacerbation of inflammation.

\section{METHODS}

It is a prospective study. A proposal for the study was sent in the prescribed format to the Institutional Ethical Committee and permission obtained.

\section{RESULTS}

\begin{tabular}{|c|c|c|c|c|c|}
\hline & CRP Day 1 & CRP Day 3 & LDH Day 1 & LDH Day 3 & RANSON \\
\hline CRP Day 1 & & $\begin{array}{c}\mathrm{r}=.937 \\
\mathrm{p} \text {-value }<.001\end{array}$ & $\begin{array}{c}\mathrm{r}=.805 \\
\mathrm{p} \text {-value }<.001\end{array}$ & $\begin{array}{c}\mathrm{r}=.844 \\
\mathrm{p} \text {-value }<.001\end{array}$ & $\begin{array}{c}r=.731 \\
p \text {-value }<.001\end{array}$ \\
\hline CRP Day 3 & & & $\begin{array}{c}r=.839 \\
\text { p-value }<.001\end{array}$ & $\begin{array}{c}\mathrm{r}=.884 \\
\text { p-value }<.001\end{array}$ & $\begin{array}{c}r=.768 \\
p \text {-value }<.001\end{array}$ \\
\hline LDH Day 1 & & & & $\begin{array}{c}\mathrm{r}=.908 \\
\text { p-value }<.001\end{array}$ & $\begin{array}{c}r=.765 \\
p \text {-value }=.027\end{array}$ \\
\hline LDH Day 3 & & & & & $\begin{array}{c}r=.754 \\
p \text {-value }<.001\end{array}$ \\
\hline \multicolumn{6}{|c|}{ Pearson's correlation coefficient $(\mathrm{r})$ used; $\mathrm{p}$-value $<0.05$ is significant; } \\
\hline
\end{tabular}
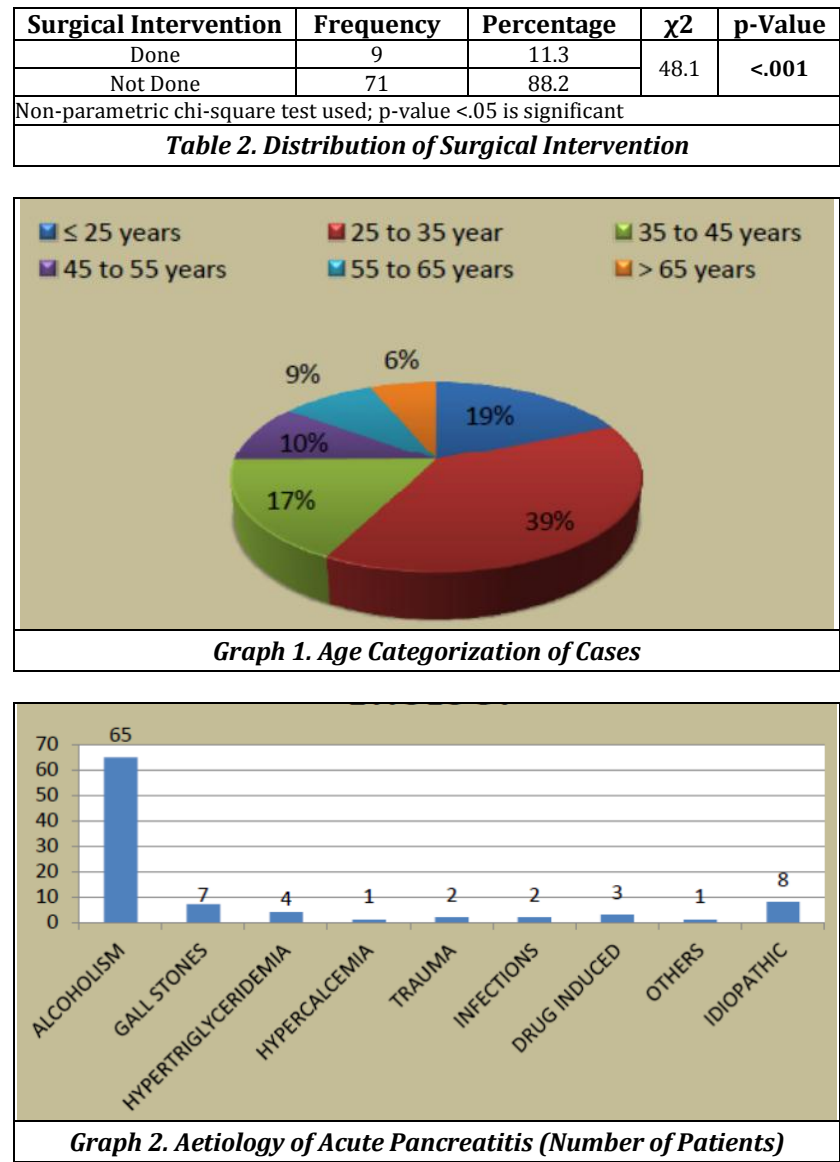

\section{Study Setting}

The study was conducted in the Department of General Surgery, S.V.R.R.G.G. Hospital, Tirupati.

\section{Inclusion Criteria}

- Patients of more than 18 years of age, both sexes and diagnosed of acute pancreatitis were included in the study.

- Acute onset of typical abdominal pain consistent with pancreatitis.

- Elevation of serum pancreatic enzymes.

- Diagnosis confirmed by imaging studies (Ultrasound or computerised tomography)

\section{Exclusion Criteria}

Patients who had chronic pancreatitis, irrespective of age and sex were excluded.

\section{Methods of Collection of Data}

- Detailed history of the cases.

- Clinical examination.

- Routine laboratory investigations.

- Relevant special investigations.

- Follow up.

\section{Investigations Done for this Study}

- Blood - Hb\%, TLC, DC, RBS, Blood Urea, Serum Creatinine, Serum Bilirubin, AST, ALT, ALP, Serum Calcium, Serum Na+, Serum K+

- Urine-Albumin, Sugar, Microscopy. 
- Radiological study- Plain X-Ray Abdomen, Chest X-Ray, USG abdomen, Endoscopic USG, CT Abdomen, MRI Abdomen.

- Specific investigation: Serum amylase, Serum lipase, Serum CRP and LDH

\section{Statistical Analysis}

Based on the data collected, the results were analysed using appropriate statistical methods. Descriptive analysis of the demographic, clinical and laboratory parameters were done. Quantitative parameters were presented as mean and standard deviation, categorical variables were presented as frequencies and percentages. Receiver operating curve (ROC) analysis was done to determine the usefulness of CRP and LDH values on day 1 and day 3 in predicting the severity of acute pancreatitis and determine the cut off value, which gives the best combination of sensitivity and specificity. The area under the ROC curve, p-value and 95\% CI were calculated. Based on the cut off levels yielded by ROC analysis, sensitivity, specificity and predictive values of each parameter in predicting severity of pancreatitis was calculated.

Males constituted $71(88.75 \%)$ of study participants with mean age of 38.3 years and the remaining 9 (11.25\%) participants were females, having a mean age of 29.4 years.

The study group had a survival rate of $92.5 \%(n=74)$, with about 6 patients (7.5\%) facing mortality.

Alcoholism history was present in majority of cases, about 65 patients (81.25\%), whereas gall stones in only about 7 patients $(8.75 \%)$.

The area under ROC curve for Amylase was 88.7\% [95\% $\mathrm{CI}=71.6 \%-99.9 \%, \mathrm{p}$-value $=.002]$. It has sensitivity and specificity of $83.3 \%$ and $93.2 \%$ respectively for serum amylase levels at a cut off value of $566.5 \mathrm{U} / \mathrm{L}$

The area under ROC curve for Lipase was 89.3\% [95\% $\mathrm{CI}=73.1 \%-99.9 \%$, p-value $=.001]$. It has sensitivity and specificity of $83.3 \%$ and $94.6 \%$ respectively for serum lipase levels at a cut off value of $808.0 \mathrm{U} / \mathrm{L}$.

The measure of the test accuracy is demonstrated by the Area under the Curve (AUC) which is 97.3\% [95\% CI= 92.1\%99.9\%, p-value <.001] for this test. The ROC curve here indicates that serum CRP levels tend to have an excellent accuracy in predicting the severity in acute pancreatitis. In addition, this prediction corresponds to a maximum sensitivity and specificity of $83.3 \%$ and $98.6 \%$ respectively for serum CRP levels on day 1 at a cut off value of $158.5 \mathrm{mg} / \mathrm{L}$.

The measure of the test accuracy is demonstrated by the Area under the Curve (AUC) which is 97\% [95\% CI=91.1\%99.9\%, p-value <.001] for this test. The ROC curve here indicates that serum CRP levels tend to have an excellent accuracy in predicting the severity in acute pancreatitis. In addition, this prediction corresponds to a maximum sensitivity and specificity of $83.3 \%$ and $98.6 \%$ respectively for serum CRP levels on day 3 at a cut off value of $165.5 \mathrm{mg} / \mathrm{L}$.

The measure of the test accuracy is demonstrated by the Area under the Curve (AUC) which is 96.3\% [95\% CI=90.4\%$99.9 \%$, p-value <.001] for this test. The ROC curve here indicates that serum LDH levels tend to have an excellent accuracy in predicting the severity in acute pancreatitis. In addition, this prediction corresponds to a maximum sensitivity and specificity of $83.3 \%$ and $98.6 \%$ respectively for serum LDH levels on day 1 at a cut off value of $743.5 \mathrm{U} / \mathrm{L}$.

The measure of the test accuracy is demonstrated by the Area under the Curve (AUC) which is 97.1\% [95\% CI= 92.5\%99.9\%, p-value <.001] for this test. The ROC curve here indicates that serum LDH levels tend to have an excellent accuracy in predicting the severity in acute pancreatitis. In addition, this prediction corresponds to a maximum sensitivity and specificity of $83.3 \%$ and $98.6 \%$ respectively for serum LDH levels on day 3 at a cut off value of $724.0 \mathrm{U} / \mathrm{L}$.

Of the 80 patients in our study, $9(11.3 \%)$ underwent surgical treatment whereas the remaining $71(88.2 \%)$ had conservative management

4 of the 9 patients (44.4\%) who underwent surgery for acute pancreatitis died. Among those who did not undergo surgery, 2 (2.8\%) died.

On an average, those who had short duration of hospital stay with a mean of around 5 days had a good survival rate $(92.5 \%)$. Those who had longer stay in hospital of 10 days average had poor survival outcome $(7.5 \%)$.

\section{DISCUSSION}

Although there has been marked decline in mortality in recent years due to acute pancreatitis, it is still a lifethreatening disease. An improved outcome in the severe form of the disease is based on early identification of the severity of disease and subsequent management of these high-risk patients. Despite the availability of several clinical (Ranson`s criteria, APACHE II score, Glasgow scoring system) and radiological scoring systems (CTSI/Balthazar scoring system), accurate prediction of the best treatment strategies and outcome after acute severe pancreatitis remains enigmatic. These scoring systems could be used as triaging tools for appropriate management.

This study was undertaken with an aim to study the role of serum CRP and serum LDH in predicting the severity of acute pancreatitis. The study period was from 25.10.2017 to 24.10.2018. 80 cases of acute pancreatitis were admitted in our hospital.

\section{Age and Sex}

In a total of 80 patients, males constituted $71(88.75 \%)$ of study participants with mean age of 38.3 years and the remaining 9 (11.25\%) participants were females, having a mean age of 29.4 years. Males: Females ratio was 7.9:1. The minimum age of the study group was 18 years and the maximum were 71 years, youngest male being 18 years and youngest female being 20 years. Majority of the patients were between the age group of 25 to 35 years (38.7\%).

\section{Aetiology}

In the present study, alcoholism history was present in majority of cases, about 65 patients $(81.25 \%)$, whereas gall stone was in only about 7 patients $(8.75 \%)$.

The most common cause in males was alcoholism, whereas in females, it was gall stone disease (Incidence of gall stones being higher in women). Idiopathic aetiology was labelled after ruling out other causes like doing serum triglycerides, calcium, antinuclear antibodies. 


\section{Prediction of Severity}

The natural history of the disease varied from mild to severe necrotising pancreatitis. Most of these patients recovered through the conservative management, but a few patients progressed to pancreatic necrosis and multi organ dysfunction. Amongst the various clinical scoring systems, the most feasible one i.e., Ranson's score was calculated and was compared with the Serum CRP and Serum LDH levels on days $1 \& 3$.

In our study, Ranson's score was ranging from a minimum of 1 to a maximum of 6.15 cases were severe as per the Ranson's score (18.75\%). Majority of the cases were mild cases $(n=65 ; 81.25 \%)$ with a Ranson's score of less than or equal to 2 . Amongst the 15 severe cases, there was a history of alcoholism in most of the cases (14 among the 15 cases). Gall stone was present in 2 of these 15 patients.

\section{Biochemical Markers \\ Serum Amylase}

The area under ROC curve for Amylase was 88.7\% [95\% CI= $71.6 \%-99.9 \%$, p-value $=.002]$. It has sensitivity and specificity of $83.3 \%$ and $93.2 \%$ respectively at a cut off value of 566.5 $\mathrm{U} / \mathrm{L}$.

\section{Serum Lipase}

The area under ROC curve for Lipase was 89.3\% [95\% $\mathrm{CI}=73.1 \%-99.9 \%$, p-value=.001]. It has sensitivity and specificity of $83.3 \%$ and $94.6 \%$ respectively at a cut off value of $808.0 \mathrm{U} / \mathrm{L}$.

According to the above two ROCs, the sensitivity and specificity of serum amylase and lipase holds good in predicting the severe form in acute pancreatitis at cut off values of $566 \mathrm{U} / \mathrm{L}$ and $808 \mathrm{U} / \mathrm{L}$ respectively. This is in contrast to literature where the amylase level usually was found to be above $1000 \mathrm{U} / \mathrm{L}$ (thrice the normal) and lipase was above $600 \mathrm{U} / \mathrm{L}$ to detect severe pancreatitis. This is not true always as seen in other literature because amylase and lipase don't have much specificity in predicting severity.

\section{Serum CRP Day 1}

The area under ROC curve for CRP on day 1 was $97.3 \%$ [95\% $\mathrm{CI}=92.1 \%-99.9 \%$, p-value $<.001]$ with a maximum sensitivity and specificity of $83.3 \%$ and $98.6 \%$ respectively for serum CRP levels on day 1 at a cut off value of $158.5 \mathrm{mg} / \mathrm{L}$.

\section{Serum CRP Day 3}

The area under ROC curve for CRP on day 3 was 97\% [95\% $\mathrm{CI}=91.1 \%-99.9 \%$, p-value $<.001]$ with a maximum sensitivity and specificity of $83.3 \%$ and $98.6 \%$ respectively for serum CRP levels on day 3 at a cut off value of $165.5 \mathrm{mg} / \mathrm{L}$.

\section{Serum LDH Day 1}

The area under ROC curve for LDH on day 1 was $96.3 \%$ [95\% $\mathrm{CI}=90.4 \%-99.9 \%$, p-value $<.001]$ with a maximum sensitivity and specificity of $83.3 \%$ and $98.6 \%$ respectively for serum LDH levels on day 1 at a cut off value of $743.5 \mathrm{U} / \mathrm{L}$.

\section{Serum LDH Day 3}

The area under ROC curve for LDH on day 3 was $97.1 \%$ [95\% $\mathrm{CI}=92.5 \%-99.9 \%$, p-value $<.001]$ with a maximum sensitivity and specificity of $83.3 \%$ and $98.6 \%$ respectively for serum LDH levels on day 3 at a cut off value of $724.0 \mathrm{U} / \mathrm{L}$.
The sensitivity and specificity of serum CRP and serum LDH in predicting severity in acute pancreatitis were calculated on days 1 and 3. Both the parameters had excellent accuracy in predicting severity in acute pancreatitis above the determined cut off values.

\section{Management and Complications}

There was no gold standard therapy for acute pancreatitis except for conservative management such as nil by mouth, IV fluids, antibiotics, proton pump inhibitors and analgesics.

Average hospital stay was 5 days in patients who had survived, higher score on Ranson's was associated with more prolonged stay about average of 10 days. The average percentage wise distribution was

9 of these patients (11.25\%) underwent surgical management. Open necrosectomy was done in 3 patients (33.3\%), 4 underwent abscess drainage (44.4\%). 3 had to go through ERCP followed by lap cholecystectomy (33.3\%).

4 of the 9 patients (44.4\%) who underwent surgery for acute pancreatitis died; this is slightly higher than the $20 \%$ $25 \%$ rate in the literature.

\section{Complications}

Most common local complication was pseudocyst of pancreas $5 \%(n=4)$ along with pancreatic abscess and pleural effusion, each $5 \%$. Splenic vein thrombosis was found in $3.75 \%(n=3)$ of the patients. Necrosis was also found in $3.75 \%(n=3)$.

\section{Mortality}

In the present study, mortality rate was $7.5 \%$ (In about 6 patients). 5 of these patients had a Ranson's score of more than 3 (Severe form). 1 of the patients had Ranson's score less than or equal to 2 (mild variety).

\section{CONCLUSIONS}

Acute pancreatitis is a condition associated with high morbidity and mortality. Early aggressive management has been shown to reduce morbidity and mortality, for which early diagnosis and assessment of severity are essential. To predict the severity of pancreatitis, before the occurrence of the multiple organ dysfunction, which usually emerges by the second or third day, numerous parameters were analysed in my study that can be detected in serum on admission. The higher the blood concentration of these parameters, the more severe was the disease. Based on the above observations, the conclusion from my study was that-

- The sensitivity and specificity of both serum CRP and LDH was excellent in predicting the severity of acute pancreatitis even on day 1 on par with traditional scoring systems.

- Both LDH and CRP are simple, easily available, economical biochemical parameters to predict the severity of acute pancreatitis in the early phase of the disease.

- They both had similar diagnostic accuracy as compared to the more widely used clinical and radiological scoring systems. These markers are also better indicators in predicting complications and further management.

\section{REFERENCES}

[1] McKay CJ, Imrie CW. The continuing challenge of early mortality in acute pancreatitis. $\mathrm{Br} \mathrm{J}$ Surg 2004;91(10):1243-4. 


\section{Jemds.com}

[2] Steinberg W, Tenner S. Acute pancreatitis. N Engl J Med 1994;330(17):1198-210.

[3] Toh SK, Phillips S, Johnson CD. A prospective audit against national standards of the presentation and management of acute pancreatitis in the South of England. Gut 2000;46(2):239-43.

[4] Lankisch PG, Schirren CA, Kunze E. Undetected fatal acute pancreatitis: Why is the disease so frequently overlooked? Am J Gastroenterol 1991;86(3):322-6.

[5] Dambrauskas Z, Giese N, Gulbinas A, et al. Different profiles of cytokine expression during mild and severe acute pancreatitis. World Journal of Gastroenterology 2010;16(15):1845-53.

[6] Abu-Zidan FM, Bonham MJD, Windsor JA. Severity of acute pancreatitis: a multivariate analysis of oxidative stress markers and modified Glasgow criteria. British Journal of Surgery 2000;87(8):1019-23.

\section{Original Research Article}

[7] Gloor B, Muller CA, Worni M, et al. Late mortality in patients with severe acute pancreatitis. British Journal of Surgery 2001;88(7):975-9.

[8] Birgisson H, Möller PH, Birgisson S, et al. Acute pancreatitis: a prospective study of its incidence, aetiology, severity and mortality in Iceland. Eur J Surg 2002;168(5):278-82.

[9] De Beaux AC, Palmer KR, Carter DC. Factors influencing morbidity and mortality in acute pancreatitis: an analysis of 279 cases. Gut 1995;37(1):121-6.

[10] Chatzicostas C, Roussomoustakaki M, Vlachonikolis IG, et al. Comparison of Ranson, APACHE II and APACHE III scoring systems in acute pancreatitis. Pancreas 2002;25(4):331-5. 